\title{
A study of the stable regions in the planetary system HD 74156 - Can it host earthlike planets in habitable zones?
}

\author{
R. Dvorak, E. Pilat-Lohinger, B. Funk, and F. Freistetter
}

Institute for Astronomy, University of Vienna, Türkenschanzstrasse 17, 1180 Vienna, Austria

Received 2 July 2003 / Accepted 11 September 2003

\begin{abstract}
Using numerical methods we thoroughly investigate dynamical stability in the region between the two planets found in HD 74156. The two planets with semimajor axes $0.28 \mathrm{AU}$ and $3.82 \mathrm{AU}$ move on quite eccentric orbits $(e=0.649$ and 0.354$)$. There is a region between 0.7 and 1.4 AU which may host additional planets which we checked via numerical integrations for different dynamical models. Besides the orbital evolution of several thousands of massless planets in a three-dimensional restricted 4-body problem (host star, two planets + massless bodies) we also have undertaken test computations of the orbital evolution of fictitious planets with masses of $0.1,0.3$ and $1 M_{\mathrm{JUP}}$ in the region between HD 74156b and HD 74156c. For direct numerical integrations up to $10^{7}$ years we used the Lie-integrator, a method with adaptive step-size. We also computed the Fast Lyapunov Indicators to detect chaotic motion in this region. We emphasize the important rôle of the inner resonances (with the outer planet) and outer resonances (with the inner planet) with test bodies located inside the resonances. In these two "resonance" regions almost no orbits survive. The region between the 1:5 outer resonance ( $0.8 \mathrm{AU})$ and the 5:1 inner resonance (1.3 AU), just in the right position for habitability, is also not very likely to host planets. Our results do not strictly "forbid" planets to move in the habitable zone, but their existence is unlikely.
\end{abstract}

Key words. stars: individual: HD 74156 - stars: planetary systems - habitable zones

\section{Introduction}

Since the discovery of the first extrasolar planetary system about 10 years ago a major point of dynamical investigations has been the determination of stable regions in extrasolar planetary systems where additional planets on stable orbits may exist. Today we have evidence for 101 planetary systems with 116 planets, where 13 systems have more than one planet. The existence of three (or more) massive bodies allows stability studies of the system itself; it is of special interest when a planet is part of a double star system like in $\gamma$ Cephei (Dvorak et al. 2003). Here we are interested in a system with two planets which could permit other planets on stable orbits. We have therefore undertaken a dynamical study with the aid of numerical experiments of the G0 star HD 74156 hosting two planets on very eccentric orbits $(e \sim 0.65$ for the inner planet HD 74156b and $e \sim 0.4$ for the outer planet HD 74156c) ${ }^{1}$. As two recent publications show - Barnes \& Raymond (2002) write "(HD 74156) contain(s) broad zones of stability" whereas Menou \& Tabachnik (2003) found no surviving planets in this region - the problem of additional planets in HD 74156 could not be answered satisfactorily up to now. Our investigation unveils the complicated structure of this region containing stable

Send offprint requests to: R. Dvorak, e-mail: dvorak@astro.univie.ac.at

1 We will use in the following the suffix "b" for the inner and "c" for the outer planet. and unstable orbits close to each other and shows that regular, chaotic and also sticky orbits exist in this domain.

In the following we discuss the dynamical models used and the method of establishing dynamical stability via longterm numerical integrations and chaos-indicators. Then we discuss the specific rôle of resonances in the region between the two planets from $0.44 \mathrm{AU}$ to $2.4 \mathrm{AU}$ caused by the inner planet (up to $0.8 \mathrm{AU}$ ) and the outer planet (down to $1.3 \mathrm{AU}$ ). Next the domain between these resonances is studied and the stability properties of possible orbits of massless planets are shown. We also placed massive planets between $0.8 \mathrm{AU}$ and $1.3 \mathrm{AU}$ (also on inclined orbits) to check their stability in this "habitable zone" (=HZ, Kasting et al. 1993).

\section{The dynamical model and the methods}

The orbital parameters were taken from the Geneva group of observers ${ }^{2}$ with $a_{\mathrm{b}}=0.28 \mathrm{AU}, e_{\mathrm{b}}=0.647$, the mass $M_{\mathrm{b}}=1.61 M_{\mathrm{Jupiter}}$ and $a_{\mathrm{c}}=3.82 \mathrm{AU}, e_{\mathrm{c}}=0.354$ and the mass $M_{\mathrm{c}}=8.21 M_{\text {Jupiter }}$.

Because of the large eccentricities of the two planets an analytical method to solve this problem does not work; any

\footnotetext{
${ }^{2}$ from obswww.unige.ch/ udry/planet/hd74156.html before the corrections of July, 16th 2003; the new large eccentricity of HD 74156b makes the existence of stable orbits in the HZ even more unlikely than stated in the conclusions.
} 
perturbation approach will fail. The methods widely used by different groups are numerical experiments in appropriate dynamical models. In our study we used the following four different models:

- the restricted 4-body problem consisting of the star, the two planets and - as test bodies - massless planets in the same plane (=Ia) and on inclined orbits $(=\mathbf{I b})$;

- the full 4 body-problem, where the additional planets had masses in the range between $0.1 M_{\mathrm{JUP}}$ to $1 M_{\mathrm{JUP}}$ on planar (=IIa) and on inclined orbits (=IIb).

Integration of the Newtonian equations of motion (the masses involved are point masses) was undertaken using the LieIntegration method which has already been extensively used by our group (e.g. Hanslmeier \& Dvorak 1984; Lichtenegger 1984). It uses an automatic step-size and is - due to the recurrence of the Lie-terms - a very fast and precise integration method.

As a second, independent tool to check the domains between the two existing planetary orbits we utilized the Fast Lyapunov Indicators (FLIs) developed by Froeschlé et al. (1997). This program uses a Bulirsch Stoer integration method and is especially adapted for distinguishing between chaotic and regular orbits via the FLIs.

The integration times were in some experiments extended up to $10^{8}$ years. Our escape (stability) criterion was such that no close encounters within the Hill sphere were allowed; such an orbit was classified as "unstable". The criterion for the FLIs is somewhat different: the time evolution of the FLIs defines clearly the orbital behaviour. The two methods were used complementarily and showed in most cases quite a good agreement of the results.

\section{The main resonance regions}

First of all we computed the domains in semimajor axes where the orbit of the inner (outer) planet does not allow stable orbits, because the respective aphelion (perihelion) distance would lead to close encounters. Thus the possible region for circular orbits without a crossing of the orbits shrinks to $0.44 \mathrm{AU} \leq$ $a \leq 1.81 \mathrm{AU}$. It is well known from the main belt of asteroids that the mean motion resonances are responsible for its structure including gaps and families. We therefore computed the main resonances up to the 1:5 mean motion resonance with the inner planet (=outer mean motion resonance - OMMR) and up to the 5:1 mean motion resonance with the outer planet (=inner mean motion resonance - IMMR). The main resonances taken into account were the major ones known from the asteroid belt: $2: 1,5: 2,7: 3,3: 1,4: 1,5: 1$ for the OMMR and the respective resonances for the IMMR (1:5, etc.). For motions in resonances the initial starting position is quite important, because sometimes a protection mechanism avoids close approaches even for large eccentricities. We have checked the IMMR and the OMMR carefully and have chosen 12 starting positions for the fictitious planet in a certain resonance with the mean anomaly $M=0^{\circ}, 30^{\circ}, \ldots, 360^{\circ}$; the respective values for two massive planets were set to $M=0^{\circ}$. The time of integration was $10^{6}$ years, but we emphasize that the extension

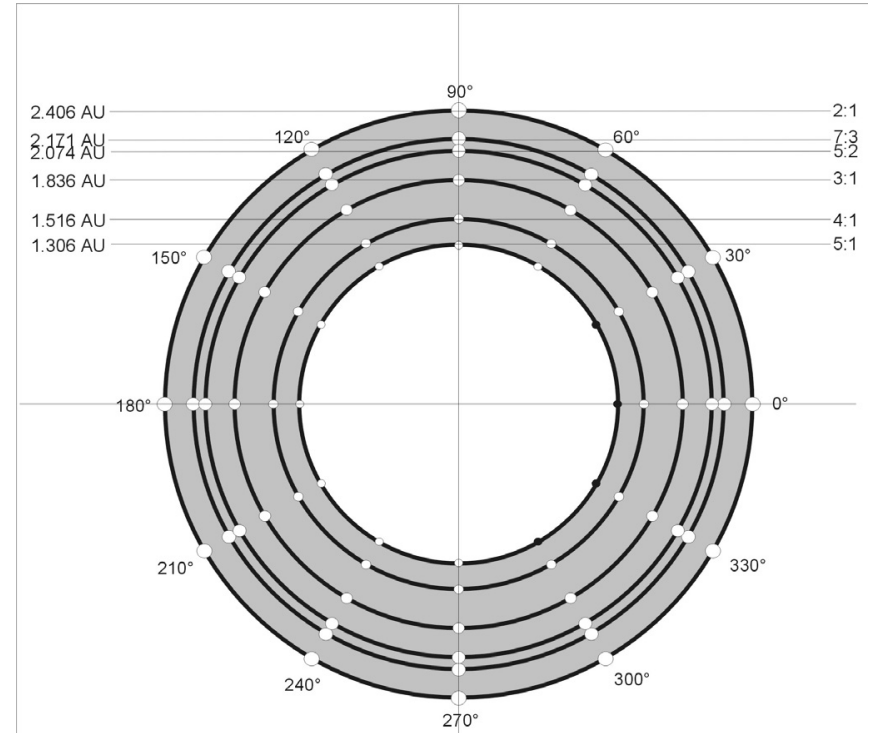

Fig. 1. Region of the IMMR in the dynamical model Ia for different values of the mean anomaly; the resonances with the outer planet (HD 74156c) are shown on the upper right side, the corresponding semimajor axes on the upper left side. Filled dark circles denote stable orbits and open circles denote unstable orbits.

from $10^{5}$ years to $10^{6}$ years changed the results only slightly; therefore we are convinced that the results are "realistic".

For the IMMR in model Ia (Fig. 1) we can see that only a few orbits survive in the 5:1 resonance; all the others suffer from close encounters with the outer planet sooner or later. We also studied the IMMR in model Ib for initial inclinations of the fictitious planet between $10^{\circ} \leq i \leq 50^{\circ}$ with $\Delta i=10^{\circ}$. Up to $i=30^{\circ}$ the stability behavior did not change significantly; for $i=40^{\circ}$ out of the 72 orbits in the IMMR only $10 \%$ survived in the higher order resonances 4:1 and 5:1.

For the OMMR in model Ia (Fig. 2) there are some orbits stable even in the 1:2 resonance quite close to the orbit of the inner planet. Their survival is due to the initial conditions which protect the fictitious planet from close encounters. The picture is different from the one for the IMMR where only resonances far away from the planet's orbit survive; for the OMMR we can see stable orbits in all resonances with the exception of the 2:5 and the 1:4 resonance. In contrast to the IMMR, the percentage of stable orbits in these resonances increases for larger initial inclinations; only for $i=50^{\circ}$ do almost no orbits survive in this region.

As another check we started different fictitious massless planets in model Ia and Ib in between the resonances using the FLIs. As an example we show the plot of an experiment where the test planet was started with $a=0.7$ AU close to the $1: 4$ resonance (Fig. $4 \mathrm{a}$ ); the starting positions of the two planets were chosen between $0^{\circ}$ and $360^{\circ}$ (model Ia); the mean anomaly of the fictitious planet was always set to $M=0^{\circ}$. Only for a strip around $M_{\mathrm{b}}=0^{\circ}$, the mean anomaly of the inner planet, do the orbits of the fictitious planet turn out to be stable; these results coincide with the stability of the 1:3 resonance of Fig. 2 for $M=0^{\circ}$ (of the fictitious planet). As a second example we 


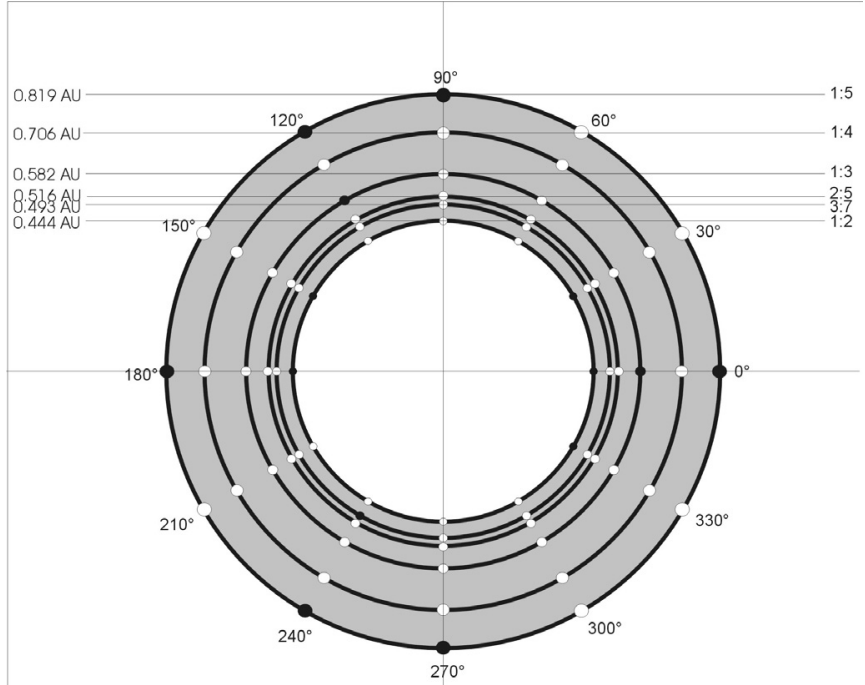

Fig. 2. Region of the OMMR in model Ia; the respective resonances are with HD 74156b. Specifications are the same as in Fig. 1.

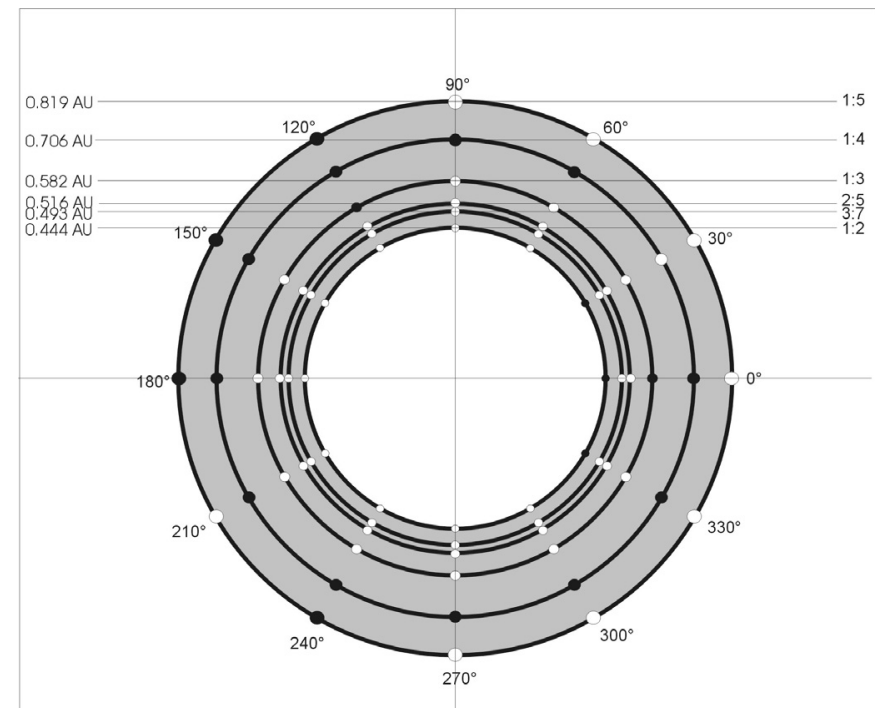

Fig. 3. Region of the OMMR in model $\mathbf{I b}\left(i=30^{\circ}\right)$; the specifications are the same as in Fig. 1.

show the dependence on the initial conditions for a fictitious planet with an initial semimajor axis $a=1.3 \mathrm{AU}$ (Fig. 4b), which is close to the 5:1 resonance. A broad strip of stable orbits is visible for certain combinations of the initial conditions, about $33 \%$ of the orbits are unstable. Here the agreement with Fig. 1 (the orbits of the 5:1 resonance at $a=1.306 \mathrm{AU}$ ) is not so good; the ratio of stable orbits to unstable ones is almost viceversa (4 out of 12 are stable). This fact shows the complicated structure of the phase space, where stable and unstable orbits are sometimes very close to each other according to high order resonances; they can be stable for quite long time on "sticky orbits" (e.g. Dvorak et al. 1998). Globally one can say that stable orbits in this domain of IMMR and the inner part of the OMMR are quite rare.

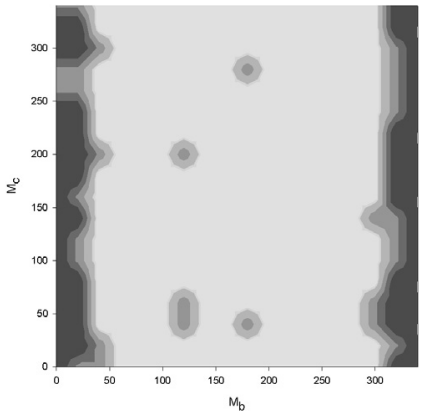

(a)

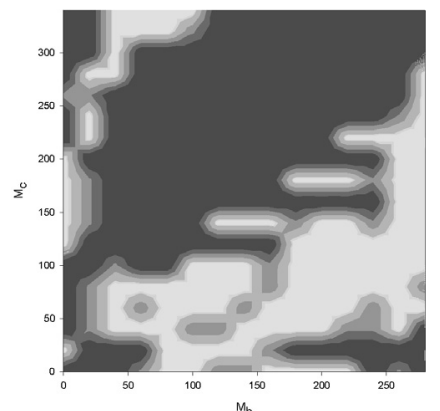

(b)
Fig. 4. a) and b): Stability of a fictitious planet started in a circular orbit with $a=0.7 \mathrm{AU}$ (left panel) and $a=1.3$ (right panel) for different initial positions of the two perturbing planets; $x$-axis is the mean anomalie of the inner planet $\left(M_{\mathrm{b}}\right), y$-axis is the mean anomalie of the outer planet $\left(M_{\mathrm{c}}\right)$. Stable regions are marked as black regions according to the values of the FLIs.

\section{The region between the resonances}

For the region between 0.8 and $1.3 \mathrm{AU}$, the $\mathrm{HZ}$, we wanted to check how the stability of the orbits there depends on the initial eccentricities of the two planets (we did not change their semimajor axes, which seem to be quite well determined through the radial velocity curves). We have therefore undertaken straightforward integrations in model Ia for four fixed values $e_{\mathrm{c}}=0.3,0.35,0.4$ and 0.45 . For each diagram one parameter was the initial semimajor axis of the fictitious planet $0.6 \mathrm{AU} \leq a \leq 1.4 \mathrm{AU}$, the other one was the eccentricity of the inner planet $0.5 \leq e_{\mathrm{b}} \leq 0.7$. The actual orbital parameters are quite well inside these ranges of eccentricities of the orbits of the two planets. Our criterion for the "grade of stability" was the largest eccentricity of the - initially circular orbit of the fictitious planet during the whole integration. This is characterized in Fig. 5 with different grey tones from dark to light grey (= escaping orbit) The criterion for the darkest region with $e<0.2$ guarantees that the differences in distance to the host star and thus the temperature changes are relatively small and favor the development and stability of atmospheres (Lammer 2003, private communication).

For $e_{\mathrm{c}}=0.3$ (Fig. 5a) - besides the "finger" around $a=$ $0.7 \mathrm{AU}$ (1:4 resonance) - we see a broad stability region between 0.9 and 1.4 AU where the eccentricities are $e<0.3$; it becomes slightly smaller for larger $e_{\mathrm{b}}$. At $a=1.25 \mathrm{AU}$, close to the $16: 3$ resonance, there exists a stable strip of orbits with small eccentricity variations $(e<0.15)$. This strip is also visible in Fig. $5 \mathrm{~b}\left(e_{\mathrm{c}}=0.35\right)$, where $e_{\mathrm{b}}=0.649$ is indicated by a straight line. In the next 2 figures (Fig. $5 \mathrm{c}$ for $e_{\mathrm{c}}=0.4$ and Fig. $5 \mathrm{~d}$ for $e_{\mathrm{c}}=0.45$ ) we see how the stability of the orbits weakens with increasing eccentricity of the outer planet. Whereas in Fig. 5c we still see a small finger of very stable orbits at $a=1.25 \mathrm{AU}$, in Fig. $5 \mathrm{~d}$ no orbits of the fictitious planets for $a \geq 1.2$ AU survive! 

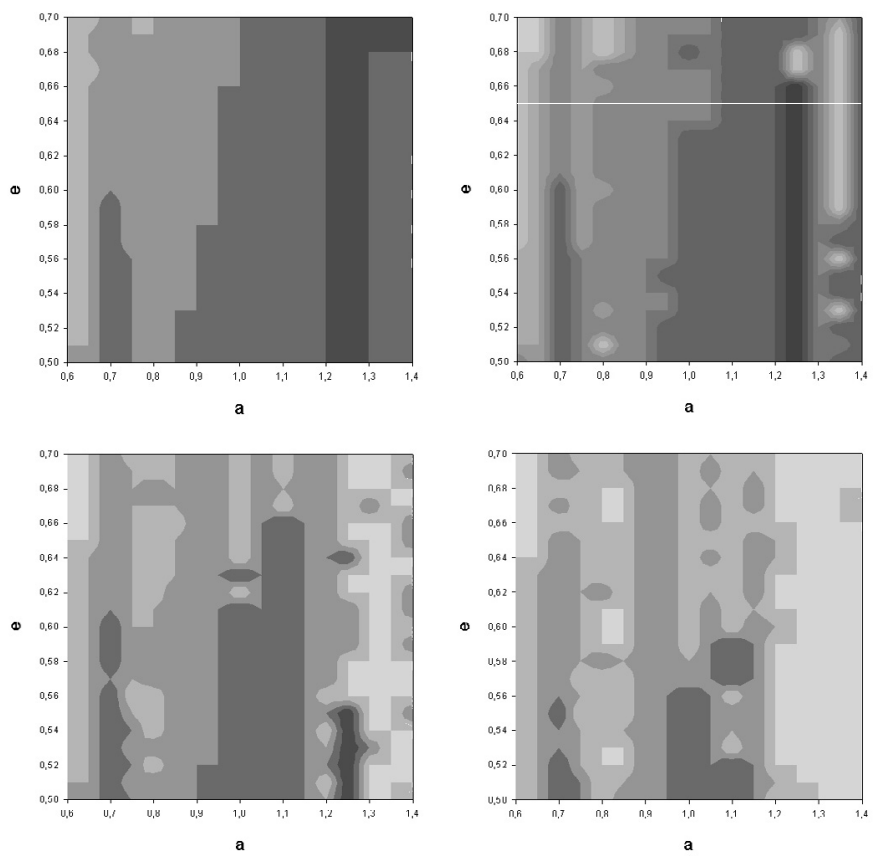

Fig. 5. Regions of motion for a fictitious planet between the inner planet and the outer planet of HD 74156 for an eccentricity $e_{\mathrm{c}}=0.3$ (a) upper left graph), $e_{\mathrm{c}}=0.35$ (b) upper right graph), $e_{\mathrm{c}}=0.4$ (c) lower left graph) and $e_{\mathrm{c}}=0.45$ (d) lower right graph) for the outer planet. Abscissa is the eccentricity of the inner planet (with an actual value of 0.649), ordinate is the initial semimajor axes of the fictitious planet always started in a circular orbit, dark regions are stable; white regions denote escaping orbits.

We also checked how secular resonances may act in this region between OMMR and $\mathrm{IMMR}^{3}$. It turned out that both planets have periods on the order of some $10^{4}$ years while for planets in the $\mathrm{HZ}$ these frequencies are in the order of only some $10^{3}$ years. We conjecture that the reason for chaotic motion between the planets $\mathrm{b}$ and $\mathrm{c}$ are the recently discovered three-body-resonances (e.g. Morbidelli 2002) which are important for asteroids in the main belt - but this will be investigated in more detail in a future study.

\section{Massive planets}

Further computations have been undertaken in model IIa and IIb for the region between IMMR and OMMR for three different masses of the additional planet, namely 0.1 , 0.3 and $1 M_{\text {JUP. }}$. The orbital character of these planets was not very different from the ones of the non-massive fictitious planets: only very few orbits were found to be stable (especially around $a=1.1 \mathrm{AU}$ ), but most of them were unstable for larger inclinations. This is somewhat in contradiction to the abstract already cited above (Barnes \& Raymond 2002). We plan a complete study, where massive planets are involved, using the Lie-code in a parallel version (Stadel et al. 2003).

\section{Conclusions}

In this study we checked how the motion in resonances destroys certain stability regions outside planet $\mathrm{b}$ (OMMR) and inside planet $\mathrm{c}$ in the extrasolar system HD 74156. Furthermore the small region between $0.8 \mathrm{AU}$ and 1.3 AU was studied through numerical experiments using the orbits of thousands of fictitious planets there. The main result is that orbits can survive there for long time intervals, but they seem to be quite rare and depend on very special initial conditions. We found orbits surviving for more than $10^{7}$ years for different initial semimajor axes, but very close to these orbits others do not survive $10^{5}$ years. This typical behavior of stickiness is well known in regions were regular and chaotic motions are mixed. For initial conditions close to $a=1.25$ AU the orbits stay within the eccentricity range $e \leq 0.12$, thus well inside the HZ of HD 74156 .

Acknowledgements. E. Pilat-Lohinger wishes to acknowledge the support by the Austrian FWF (Hertha Firnberg Project T122). B. Funk and F. Freistetter wish to acknowledge the support by the Austrian FWF (Project P14375-TPH). Special thanks go to Markus Gyergyovits, who drew our attention to this problem and Ruth Grützbauch, who helped editing this article. We also thank James Howard for improving the language.

\section{References}

Barnes, R. K., \& Raymond, S. N. 2002, AAS, 46.15

Dvorak, R., Contopoulos, G., Efthymiopoulos, Ch., \& Voglis, N. 1998, PS\&S, 46, 1567

Dvorak, R., Pilat-Lohinger, E., Funk, B., \& Freistetter, F. 2003, A\&A, 398, L1

Froeschlé, C., Lega, E., \& Gonczi, R. 1997, CMDA, 67, 41

Hanslmeier, A., \& Dvorak, R. 1984, A\&A, 132, 203

Kasting, J. F., Whitmire, D. P., \& Reynolds, R. T. 1993, Icarus, 101, 108

Lichtenegger, H. 1984, CMDA, 34, 357

Menou, K., \& Tabachnik, S. 2003, ApJ, 583, 473

Morbidelli, A. 2002, Modern celestial mechanics: Aspects of solar system dynamics (London: Taylor \& Francis), $231 \mathrm{ff}$

Stadel, J., Freistetter, F., \& Dvorak, R. 2003 (in preparation)

${ }^{3}$ Secular resonances arise when the motion of the perihelion (Keplerian element $\omega$ ) or the motion of the longitude of the node (Keplerian element $\tilde{\omega}$ ) is in resonance with the respective motion of the regarded planet. 